\title{
Résilience du secteur alimentaire face à la crise Covid-19 et perspectives pour les politiques agricoles en République du Congo
}

\author{
Junior Bitsoumanou Nkounkou ${ }^{1, *}$ et Ludovic Temple ${ }^{2,3}$ \\ ${ }^{1}$ Section Programme - Suivi et évaluation des projets/programmes, FAO Congo, 14, Rue Behagle - Centre-Ville, BP 972 Brazzaville, \\ République du Congo \\ 2 CIRAD, UMR INNOVATION, F-34398 Montpellier, France \\ ${ }^{3}$ INNOVATION, Univ Montpellier, CIRAD, INRAE, Institut Agro, Montpellier, France
}

\begin{abstract}
Résumé - La crise économique et sanitaire due à la Covid-19 interpelle les trajectoires de reconfiguration des systèmes alimentaires en République du Congo. Elle questionne la capacité des mesures de régulation prises par les pouvoirs publics et des stratégies d'adaptation des acteurs à induire des inflexions dans la gouvernance structurelle du système alimentaire du pays. L'article analyse les stratégies d'adaptation à la crise en mobilisant des données secondaires et des expériences d'accompagnement de politiques publiques agricoles et alimentaires conduites par les auteurs. Au Congo, le secteur alimentaire reste tributaire des importations et repose sur une agriculture vivrière extensive. Pour gérer la pandémie, un confinement général « flexible » de la population a été appliqué. Les achats de panique, la réduction de la durée d'activité des marchés et la hausse temporaire des prix des produits alimentaires ont révélé les fragilités du secteur, liées à sa dépendance aux marchés internationaux. La crise a cependant confirmé la capacité d'innovation collective du secteur informel. Elle réhabilite la reconnaissance d'une agriculture de proximité, source d'emplois pour des populations vulnérables. Elle interpelle la conception de politiques agricoles et alimentaires futures au regard d'enjeux d'intégration des risques sanitaires, alimentaires et sociaux, dans le contexte du bassin forestier du Congo, qui est exposé à des risques de résurgence de zoonoses existantes et d'arrivée de maladies émergentes.
\end{abstract}

Mots clés : approvisionnement / Congo / Covid-19 / sécurisation alimentaire / innovation

\begin{abstract}
Resilience of the food sector in the face of the Covid-19 crisis and prospects for agricultural policies in the Republic of Congo. The economic and health crisis caused by COVID-19 calls into question the reconfiguration trajectories of food systems in the Republic of Congo. It questions the extent to which the regulatory measures taken by the public authorities and the adaptation strategies of the actors lead to changes in the structural governance of the country's food system. The article analyses the strategies for adapting to the crisis, by using secondary data and authors' experience of support to public policies in the agricultural and food sector. In Congo, the food sector remains dependent on the outside world and relies on extensive subsistence farming. To manage the pandemic, a general, 'flexible' containment of the population has been applied. Panic buying, reduced market activities and the temporary rise in food prices revealed the fragility of the sector linked to its dependence on international markets. However, the crisis has confirmed the collective innovative capacity of the informal sector. It has restored the recognition of urban agriculture has source of employment for vulnerable populations. It calls into question the design of future agricultural and food policies with regard to the integration of health, food and social risks, in the context of the Congo Forest Basin, which is exposed to the risk of a resurgence of existing zoonoses and emerging diseases.
\end{abstract}

Keywords: supply / Congo / COVID-19 / food security / innovation

\footnotetext{
*Auteur de correspondance : junior.bitsoumanou@fao.org
} 


\section{Introduction}

La pandémie de la Covid-19 a créé une situation d'incertitude socio-économique qui interpelle la capacité des politiques publiques à permettre aux populations les plus vulnérables de s'adapter aux différentes urgences et à structurer des orientations qui réduisent les probabilités d'émergence des crises sanitaires de ce type. Considérant sa forte croissance démographique, l'Afrique concentre un grand nombre de situations d'insécurité alimentaire. Les liens de causalité entre la crise sanitaire, l'insécurité alimentaire et l'adéquation des politiques publiques sont donc aujourd'hui au centre des préoccupations des décideurs politiques de ce continent. Le débat sur la gouvernance structurelle des politiques de sécurité alimentaire en Afrique a fait l'objet de nombreux travaux. Ainsi, Bricas et al. (2016) montrent comment la dépendance alimentaire a été renforcée dans ces pays par les politiques d'ajustement structurel mises en place dans les années 1980, en encourageant la libéralisation des échanges agricoles. La crise sanitaire actuelle impose de répondre simultanément à l'urgence de bien gérer les relations entre la crise sanitaire et l'émergence possible de l'insécurité alimentaire, et au besoin de mettre en place des orientations stratégiques pour renforcer la résilience des systèmes alimentaires à de nouveaux chocs potentiels dans le « monde d'après » (Courleux, 2020). À travers une analyse de la littérature récente sur la crise sanitaire en Afrique sub-saharienne (Dury et al., 2021; Workie et al., 2020), et en mobilisant une recherche finalisée menée récemment en République du Congo, nous questionnons la façon dont le secteur agricole et alimentaire résiste à cette crise sanitaire et la possibilité pour les politiques de capitaliser sur la capacité de résistance de ce secteur pour définir leurs futures orientations stratégiques.

Sur le plan méthodologique, nous proposons de caractériser trois variables qui structurent potentiellement les capacités de résistance du secteur agricole et alimentaire :

- Les spécificités des contextes macro-économiques et socioécologiques nationaux et leurs dynamiques historiques;

- la capacité endogène des territoires à produire et rendre disponibles les ressources alimentaires;

- l'importance du secteur agro-artisanal et de l'économie domestique (qualifié fréquemment d'informel) pour la résilience du système alimentaire (Vonthron et al., 2016).

Notre cadre d'analyse propose de qualifier la résistance (une dimension de la résilience) du secteur alimentaire (Vonthron et al., 2016) au regard des articulations qui font interagir le secteur informel national, les importations alimentaires et les mesures de gouvernance prises par les pouvoirs publics en vue de juguler la crise liée à la Covid-19. Concernant les politiques publiques, l'analyse fait le distinguo entre les actions de court terme visant à atténuer les conditions d'exposition de la population; celles de moyen terme pour assurer plus durablement les conditions d'accès aux ressources alimentaires; et les éléments de long terme pour corriger les causes de la pandémie.

L'Afrique sub-saharienne présente une diversité de situations selon l'échelle «normée» du développement (pays émergent, à revenu intermédiaire, en voie de développement, moins avancé) et en particulier de situations géopolitiques (nature et importance des conflits, niveau de précarité, disponibilité et qualité des ressources naturelles et des ressources publiques). En Afrique centrale, les pays où les ressources pétrolières et minières pilotent l'action publique sont fréquents. Ces pays présentent aussi une situation socioécologique spécifique liée aux forêts (dense et humide) du bassin du Congo qui est un réservoir écologique important de la biodiversité mondiale.

\section{Contexte et cadres méthodologiques}

En République du Congo, la disponibilité alimentaire, notamment dans les villes qui concentrent plus de $60 \%$ de la population (INS, 2015), est très dépendante des marchés extérieurs. La valeur importante du coût annuel per capita des importations alimentaires est évaluée à $490 €$ en moyenne, de 2010 à 2019 (Fig. 1). Selon la Direction générale des douanes et droits indirects, la valeur moyenne des importations des produits agricoles et alimentaires des quatre années précédant l'arrivée de la Covid-19 est de 502 millions d'euros par an. Le programme national d'investissement agricole et de sécurité alimentaire et nutritionnelle 2017-2021 estime satisfaisante la couverture des besoins de la consommation intérieure par la production nationale pour le manioc $(95 \%)$, la banane plantain $(125 \%)$ et l'arachide $(124 \%)$. Mais il ressort un déficit en productions animales très élevé pour la viande de volaille (99\% de la consommation sont importés), la viande bovine $(99 \%)$, la viande porcine $(89 \%)$, le lait et les produits laitiers $(99 \%)$, et les produits halieutiques $(40 \%)$. Ce qui justifie les politiques agricoles et alimentaires privilégiées par l'État dans le passé, basées sur des importations en aliments mais aussi en intrants, en variétés améliorées et des transferts de technologies susceptibles d'accroître ces productions.

Les premiers travaux empiriques exploratoires sur la Covid-19 et les systèmes alimentaires mettent en relief non seulement des problèmes de disponibilité des produits pour l'approvisionnement des chaînes alimentaires (Bitsoumanou et Martin, 2020; Mialoundama et al., 2020), mais aussi des problèmes d'accessibilité en relation avec les restrictions liées à la circulation des personnes et des biens et aux autres mesures de confinement qui ont impacté les marchés urbains (FAO, 2020) durant les périodes de confinement. Ces impacts touchent particulièrement les activités du secteur informel, qui concentre près de $80 \%$ de l'emploi au Congo. Selon Sene (2020), les catégories sociales impliquées dans ce secteur sont les plus vulnérables face à la crise sanitaire de la Covid-19. La population congolaise est estimée à près de 5,5 millions d'habitants avec $44 \%$ de la population vivant en zones rurales (INS, 2015). La part de la population agricole dans la population active totale est de $37 \%$. En zone rurale, $69 \%$ des personnes sont considérées comme pauvres. Les zones rurales et Brazzaville représentent ensemble $70 \%$ de la population et abritent $77 \%$ des personnes pauvres. En l'absence de politiques ou d'institutions de protection sociale permettant de faire face aux incertitudes sanitaires, nous faisons l'hypothèse que le secteur informel (rural et urbain) est au centre des mécanismes d'adaptation permettant de surmonter la situation de crise actuelle, comme cela a été observé en Tunisie (Elloumi, 2020).

L'analyse empirique est essentielle au Congo, en raison d'un appareil statistique qui ne facilite pas l'accès aux données (Favrot et Dorier, 2018) et qui connaît une irrégularité de réalisation des grandes enquêtes, y compris agricoles. De ce fait, cet article mobilise des bases d'informations et de données 


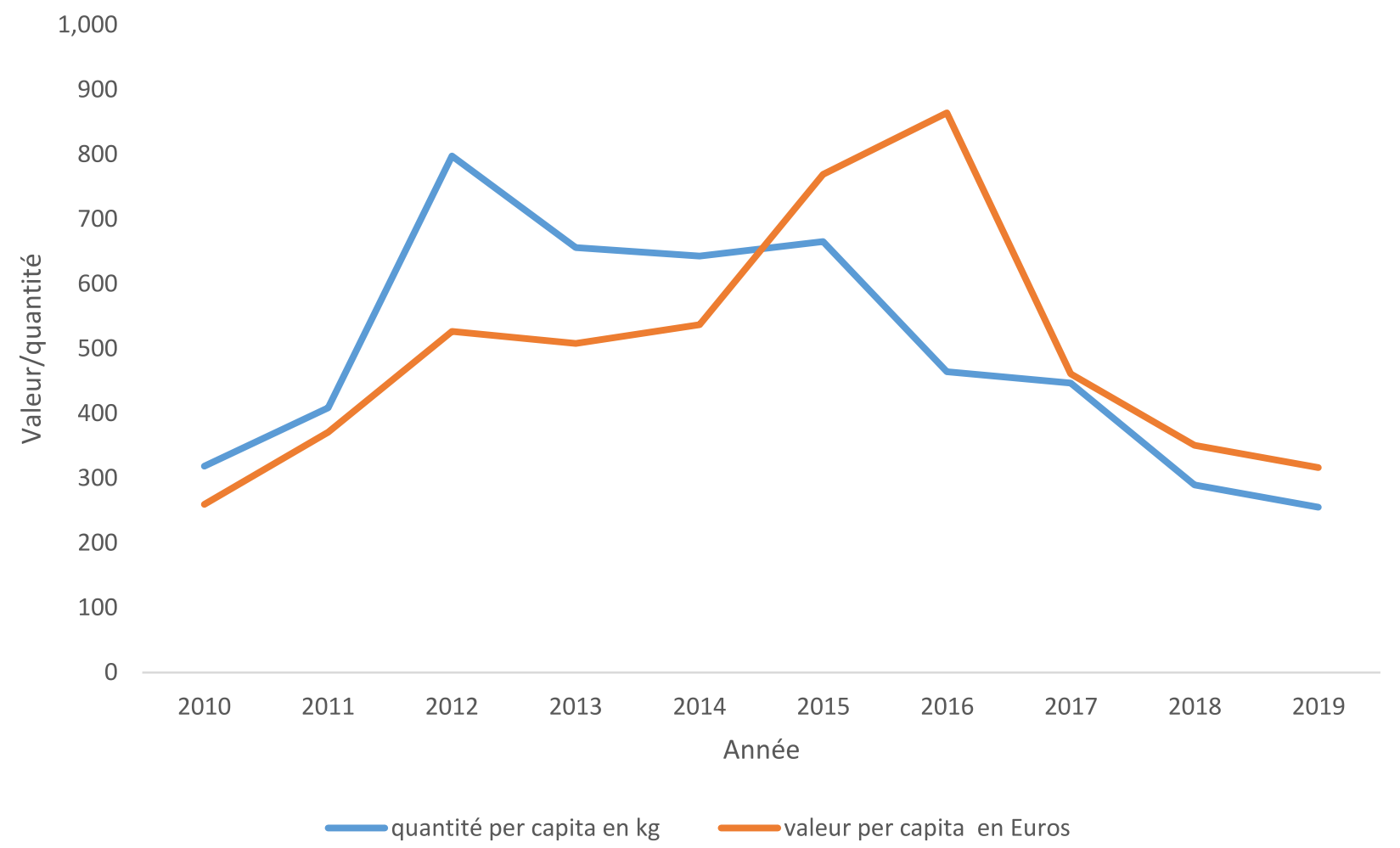

Fig. 1. Valeur et quantité des importations alimentaires par habitant et par an au Congo. Source: Institut National de la Statistique (INS).

Fig. 1. Value and quantity of food imports per capita per year in Congo.

secondaires issues des administrations congolaises et des agences de développement spécialisées dans l'alimentation et l'agriculture (PAM - Programme alimentaire mondial - et FAO - Organisation des Nations unies pour l'agriculture et l'alimentation). Il s'appuie également sur notre expérience de l'analyse des politiques publiques alimentaires dans différents contextes (Bitsoumanou et Martin, 2020; Temple et al., 2015).

\section{Caractérisation macro-économique du secteur agricole du Congo en rapport avec la rente pétrolière et émergence des maladies virales}

Du fait de ses caractéristiques édapho-climatiques et de sa superficie agricole utile de plus de 10 millions d'hectares, le Congo est reconnu comme un territoire à forte vocation agricole et forestière. De plus, ce pays du bassin du Congo abrite un réservoir de biodiversité parmi les plus importants de la planète. L'histoire post-Indépendance de l'agriculture congolaise peut être analysée à travers quatre périodes (Feintrenie, 2014). De 1960 à 1974 : Le jeune État nationalise les anciennes entreprises coloniales et crée des offices de commercialisation des produits agricoles. De 1974 à 1991 : l'État «producteur» va utiliser les recettes pétrolières pour créer des fermes d'État et 3 offices de commercialisation des cultures vivrières, du cacao et du tabac, qui garantissent un prix plancher et des débouchés aux agriculteurs. De 1991 à 1993, une courte période de libéralisation économique conduit à la liquidation des offices. De 1993 à 2000, une période d'instabilité politique et militaire qui aboutit en 2000 à un «chaos total», caractérisée par la destruction des infrastructures (routes, fermes, écoles, universités...) (Mfoukou-Ntsakala et al., 2006). De 2000 à 2020, une période de reprise démocratique et économique permet la mise en œuvre de nombreux projets et programmes agricoles et l'apparition d'investisseurs agro-industriels privés nationaux et étrangers.

Depuis les années 2000, l'anticipation d'un épuisement à venir des revenus pétroliers suscite une remise en question des politiques publiques, ce qui a conduit à la priorisation du secteur agricole dans le programme de diversification économique du Programme national de développement 2018-2022. Cependant, les revenus pétroliers assurent encore près de $80 \%$ du budget de l'État et ce dernier n'a encore eu que peu d'effets sur le secteur agricole et alimentaire. Les dix principaux produits d'exportation du Congo sont non alimentaires, avec en premier lieu le pétrole $(85 \%)$, suivi du bois brut $(4,5 \%)$. Par contre, sept des dix principaux produits importés sont alimentaires, avec au premier rang les viandes et abats comestibles $(5,8 \%)$, suivis des céréales pour l'alimentation humaine (3\%) (INS, 2021).

Sur le plan sanitaire, le programme de lutte contre les menaces pandémiques émergentes mené au Congo de 2009 à 2019 a permis de détecter chez les chauves-souris deux coronavirus connus et préoccupants pour la santé publique à l'instar du SRAS (syndrome respiratoire aigu sévère) et du MERS (syndrome respiratoire du Moyen-Orient), ainsi que neuf coronavirus inconnus. La République démocratique du Congo (RDC) a déclaré, le $1^{\mathrm{er}}$ juin 2020, la $11^{\mathrm{e}}$ épidémie de la maladie à virus Ebola (MVE). Jusqu'au 17 novembre 2020, on compte 119 cas confirmés dans la province de l'Équateur selon le tableau de bord de l'OMS sur le suivi de la MVE en RDC. 
Cette épidémie constitue une menace pour le Congo voisin, sachant que trois de ses départements (Cuvette, Plateau, Likouala), situés au nord du pays et frontaliers de la RDC, présentent des environnements favorables à la propagation rapide de cet agent pathogène.

\section{Impacts de la Covid-19 sur le secteur agricole et alimentaire}

Le confinement général de la population, qui a pris effet le 31 mars 2020 au Congo et a duré 46 jours, a suscité des protestations des maraîchers urbains et péri-urbains qui n'ont pas arrêté de produire mais ont rencontré des difficultés pour vendre leur production de légumes. Ces producteurs avaient besoin de vendre ces produits périssables pour s'assurer un minimum de revenu et la population urbaine avait besoin de produits frais pour se nourrir. Il s'en est suivi, au sein du ministère de l'Agriculture, de l'Élevage et de la Pêche, l'organisation de réunions (État, secteur privé et société civile) pour évaluer les impacts pressentis du confinement, la disponibilité des stocks alimentaires et les mesures à prendre. L'état d'urgence sanitaire a été reconduit 22 fois jusqu'au 15 juin 2021 (Encadré 1).

Encadré 1. Gouvernance publique de la crise sanitaire liée à la Covid-19

Le Gouvernement de la République du Congo a confirmé son premier cas importé de Covid-19 le 14 mars 2020. Afin de contenir la propagation du virus, notamment dans les villes de Brazzaville et de PointeNoire, qui présentent les plus fortes densités de population, le gouvernement a graduellement mis en œuvre les mesures suivantes :

- le 18 mars 2020, la fermeture des établissements scolaires et des restaurants et l'interdiction de toutes réunions/rassemblements de plus de 50 personnes sur tout le pays ;

- le 21 mars 2020, la fermeture des frontières terrestres, fluviales, maritimes et aériennes en autorisant uniquement le passage des navires et avions cargos;

- le 31 mars 2020, la déclaration de l'état d'urgence sanitaire pour une durée de 20 jours sur toute l'étendue du territoire national, couplée à l'annonce d'un confinement général ;

- le $1^{\mathrm{er}}$ avril 2020, l'autorisation de circulation pour les biens et services indispensables: produits alimentaires et boissons, charbon de bois, bois de chauffe, gazinières, réchauds, matières et intrants agricoles;

- le 6 avril 2020, la délivrance gratuite d'attestations de déplacement aux opérateurs des filières agropastorales et alimentaires;

- le 7 avril 2020, l'ouverture des marchés domaniaux, c'est-à-dire gérés par les municipalités, 3 jours par semaine (lundi, mercredi et vendredi) de 6 à $14 \mathrm{~h}$, les jours fermés étant consacrés à la désinfection des marchés. C'est pendant les jours ouvrés que la population avait le droit de circuler jusqu'à l'heure du couvre-feu ( $20 \mathrm{~h}$ à $05 \mathrm{~h}$ du matin).
Globalement, la production végétale urbaine et périurbaine du Congo n'a pas connu de grandes perturbations. L'offre en fruits et légumes ainsi qu'en autres denrées alimentaires locales a été assurée sur les marchés domaniaux pendant le confinement de la population (Bitsoumanou et Martin, 2020). C'est une des conséquences des mesures favorisant la circulation des biens alimentaires, à la fois par les acteurs des secteurs formel et informel, prises par le gouvernement en considération du poids socio-économique du secteur informel au Congo (Encadré 1).

Avec moins de 3 millions d'habitants chacune, Brazzaville et Pointe-Noire sont considérées au niveau mondial comme des villes de taille moyenne et non des mégalopoles (FAO, 2020). Leurs circuits d'approvisionnement alimentaire ont été particulièrement sollicités du fait des contraintes de circulation des personnes imposées par la crise Covid et de leur totale dépendance alimentaire, notamment pour les aliments locaux de base (manioc et banane plantain), provenant de la zone rurale. Le premier effet de la mesure de confinement a été la constitution de stocks alimentaires par la population de ces deux villes. Ce qui a conduit à l'accroissement des quantités achetées et a entraîné un mouvement de spéculation pour des denrées alimentaires (Bitsoumanou et Martin, 2020; FAO, 2020; Murhula et al., 2021). Au mois de mars 2020, cette évolution a été observée pour les aliments locaux et importés, avant d'enregistrer une baisse quasi-uniforme des prix de l'ensemble des produits alimentaires au mois d'avril 2020 (Fig. 2), suite à l'intervention du gouvernement. Cette variation, sauf pour la farine de manioc qui a gardé son prix constant au mois d'avril 2020, n'avait pas été observée durant les mois de mars et avril des années 2016 à 2019. Durant le confinement, la réduction de la durée (nombre de jours et heures d'ouverture) des activités des marchés domaniaux, couplée à l'absence d'infrastructures de stockage et de conservation, a engendré d'importantes pertes et des gaspillages de certains produits agricoles comme les fruits et légumes (bananes, oranges, citrons, litchis, tomates...) venant des zones rurales (Pool, Bouenza, Niari et Plateaux).

La viande de brousse (issue de la chasse), importante source de protéines animales des écosystèmes forestiers congolais, est fortement ancrée dans les habitudes alimentaires des ménages brazzavillois (Ofouémé-Berton, 1993) et les 35,7 tonnes de ce produit carné commercialisées à Brazzaville chaque mois (Mbété et al., 2011) proviennent principalement de départements assez éloignés de cette ville. Les mesures de restriction aux déplacements et la médiatisation faite sur le rôle des espèces animales sauvages dans l'émergence de la Covid-19 ont particulièrement réduit l'approvisionnement en viande de brousse (Mialoundama et al., 2020). Par contre, dans les zones rurales, la proximité des zones de production ainsi que les chaînes d'approvisionnement plus courtes rendent les petites villes et les villages moins vulnérables aux perturbations des réseaux de distribution des produits alimentaires locaux.

\section{Résilience et défaillance de l'économie alimentaire}

La gouvernance de la crise sanitaire par l'État a conduit à classer les activités économiques en activités « essentielles» 


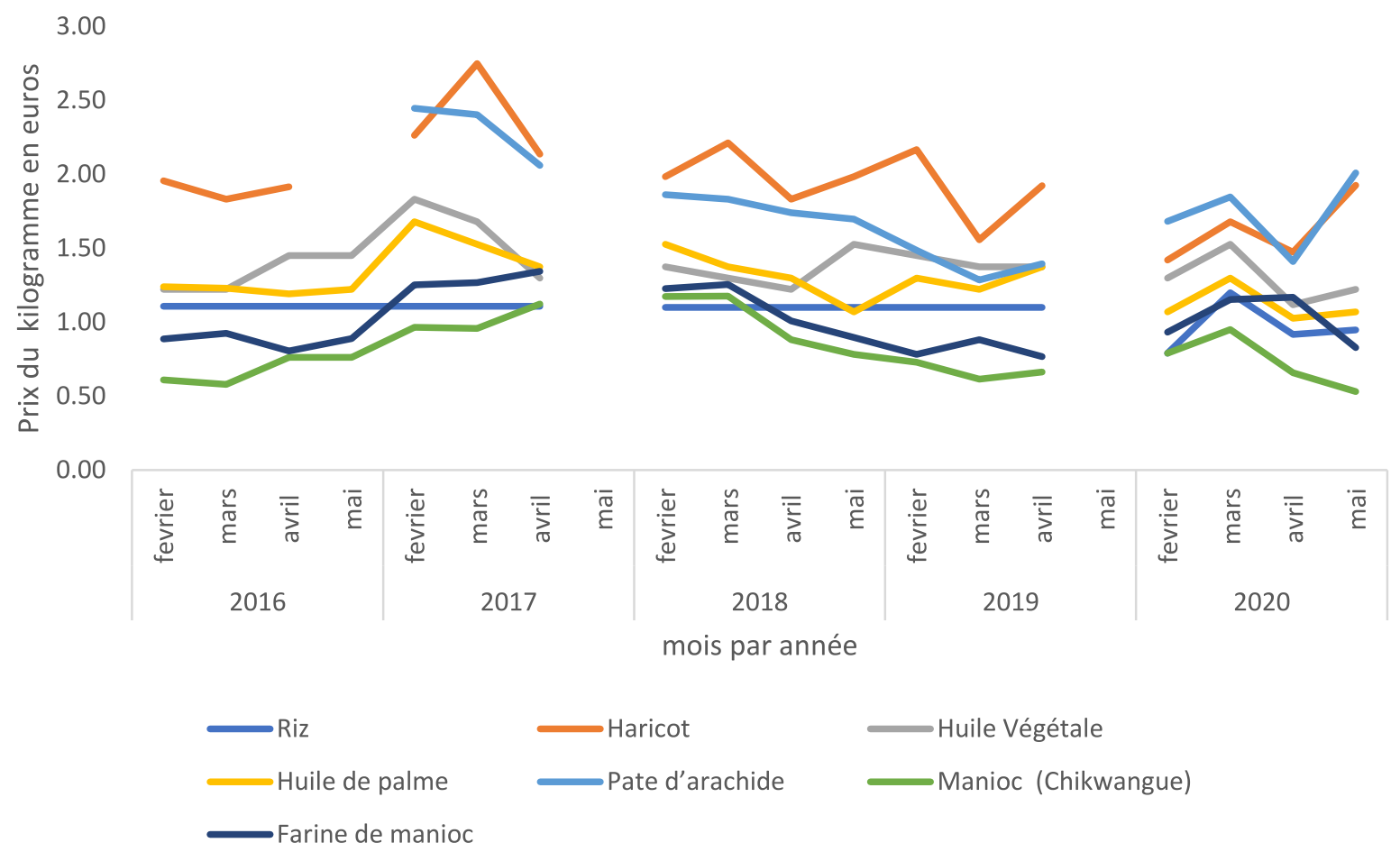

Fig. 2. Évolution des prix en euros par kilogramme des produits alimentaires durant le premier trimestre de 2016 à 2019 sur les marchés de Brazzaville. Source: ministère du Commerce, des Approvisionnements et de la Consommation et PAM Congo, suivi des prix des marchés de Brazzaville.

Fig. 2. Evolution of prices in euros per kilogram of food during the first quarter of 2016 to 2019 in the markets of Brazzaville City.

ou «non essentielles» pour limiter l'exposition et la propagation du virus. Les acteurs des activités informelles «essentielles», comme le commerce et la transformation alimentaire et l'agriculture, ont été autorisés à les poursuivre ; les activités «non essentielles » (coiffure, couture, mécanique auto, menuiserie, froid, blanchisserie, vulcanisation, restauration, chauffeurs de bus et de taxis) n'ont pas été autorisées durant le confinement. Ainsi, la majorité des acteurs du secteur alimentaire informel péri-urbain, qui concerne des populations défavorisées (production, commerce et transport de vivriers...), ont $\mathrm{pu}$ maintenir leur revenu et leur approvisionnement alimentaire. Selon le rapport du Système des Nations unies au Congo (Nations unies, 2020) sur l'évaluation des impacts socioéconomiques de la pandémie de Covid-19, ce revenu a été estimé à 53 euros pour toute la période de confinement général (46 jours) soit $1,15 €$ /jour.

Trois cent quarante trois entreprises à activités «essentielles » membres de l'Union patronale et interprofessionnelle ont connu une baisse d'activités allant de 34 à $76 \%$. La baisse de chiffre d'affaires la plus importante (jusqu'à - $64 \%$ ) concerne les entreprises de l'agroalimentaire du fait du manque d'emballages importés (bouteilles, bouchons de bouteilles, bocaux et autres) et celles de production d'aliments du bétail par manque de matières premières importées. Dans le secteur agropastoral, la filière avicole a été la plus impactée en raison de sa forte dépendance aux intrants venant de l'extérieur (poussins d'un jour, maïs, tourteaux de soja, concentrés minéralisés azotés et vitaminées, et produits vétérinaires). La dépendance aux intrants importés, liée aux circuits d'approvisionnements longs, a causé la réduction ou l'arrêt de production des entités du secteur agroalimentaire concernées.
Les importations alimentaires, également dépendantes de circuits longs, ont connu une réduction de volume au cours de l'année 2020 en comparaison avec les années 2011 à 2019. En valeur, les produits carnés sont les produits alimentaires les plus importés au Congo (INS, 2021) et les principaux exportateurs sont les pays américains et européens (Fig. 3). L'évolution de la pandémie de Covid-19 sur ces deux continents a perturbé le régime de ces importations au Congo, avec une nette incidence sur le volume annuel. Selon l'INS (2021), l'évolution globale des importations de produits carnés présentait une tendance croissante de 2018 à 2020.Elle a connu une hausse considérable au premier trimestre 2020, qui correspond à la période précédant la déclaration du confinement (31 mars 2020), suivie d'une baisse aux deuxième $(-5,3 \%)$ et troisième $(-13,8 \%)$ trimestres, qui correspondent à la période du confinement, pour rebondir au quatrième trimestre $(+24,2 \%) 2020$.

\section{Contexte de la Covid-19: facteurs d'innovation et d'adaptation}

La faible densité de peuplement des zones rurales congolaises ( 12 habitants par $\mathrm{km}^{2}$ ) est considérée comme une contrainte pour le développement agricole. Mais face à la Covid-19, elle s'est révélée comme un facteur de résilience sanitaire, car propice aux mesures de distanciation physique ; la main-d'œuvre agricole n'a quasiment pas été malade et a pu continuer son travail de production. Ce qui corrobore les travaux de Lepira et al. (2020). En dépit des difficultés de transport et des «tracasseries policières», les flux de biens 


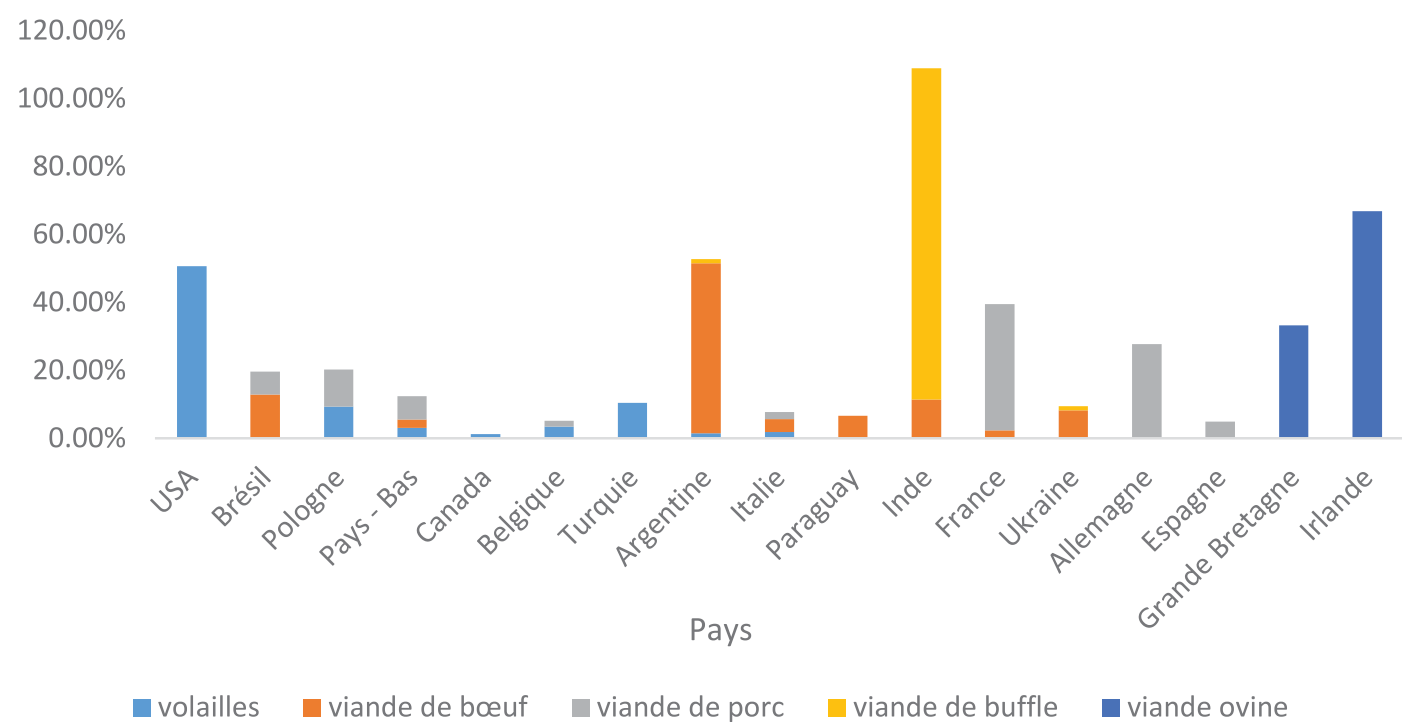

Fig. 3. Origine des principaux produits carnés importés en pourcentage par pays. Source: Direction générale de l'élevage (modifié). Fig. 3. Origin of the main imported meat products by country in percentage.

alimentaires ont été peu perturbés et les acteurs des systèmes alimentaires se sont adaptés aux contraintes du confinement (Bitsoumanou et Martin, 2020). Ils ont su innover en réactivant des modes de transport et de distribution alternatifs pour faire face au recul des activités de certains acteurs. Ainsi, les transports habituels en camions ou pick-up ont laissé la place aux motocyclettes et les marchés domaniaux fermés ont été remplacés par des vendeurs ambulants, de nouveaux petits marchés informels, le commerce de rue et la vente en ligne. Suite à l'arrêt des importations d'emballages alimentaires (bouteilles, bocaux, bouchons, papiers kraft...), les unités agro-artisanales de production de jus à base de fruits locaux ont davantage valorisé «l'économie circulaire», en recyclant les contenants des boissons importées et commercialisées par les brasseries congolaises, de façon à maintenir leur production.

Cette réactivité repose d'une part sur l'usage d'outils numériques comme les applications de vente en ligne, le mobile money (paiement mobile) et le e-food (nourriture commandée sur internet), notamment dans les villes de Brazzaville et Pointe-Noire, et d'autre part sur l'expérience du vécu des crises qui ont affecté les systèmes d'approvisionnement alimentaires par le passé, à l'instar des guerres civiles des années 1990. La période de confinement liée à la Covid-19 révèle l'agilité du secteur alimentaire qui a été aussi favorisé par des mesures de gouvernance réalistes et flexibles (Bitsoumanou et Martin, 2020). Pour limiter les impacts socio-économiques de la crise, le gouvernement a mis en place un Fonds national de solidarité, doté de plus de 150 millions d'euros selon le Journal officiel, pour renforcer le système de santé publique, soutenir les entreprises, compenser les pertes de revenu des actifs et aider les personnes vulnérables. La société civile, les entreprises et les acteurs de développement ont également apporté des aides, en nature (aliments) ou en espèces, aux personnes vulnérables : vieillards, orphelins et ménages démunis (Bitsoumanou et Martin, 2020).

\section{Quels modèles de développement agricoles et alimentaires pour l'avenir du Congo?}

Malgré les spécificités macro-économiques et écologiques du Congo (fortes importations alimentaires, rente pétrolière, existence d'écosystèmes forestiers humides et besoin de préserver la biodiversité), ses politiques de développement agricole ont jusque-là privilégié le modèle d'une agriculture industrielle intensive en intrants et en capitaux en vue d'atteindre la souveraineté alimentaire. Vu la situation de crise créée par la Covid-19, la pertinence de ce choix est questionnable à deux niveaux.

Le premier niveau interroge la vulnérabilité des agriculteurs du fait de leur dépendance structurelle à des intrants (semences, engrais, pesticides, jeunes animaux) et à des technologies (pièces de rechange, compétences) qui dépendent de pays étrangers pouvant eux-aussi être paralysés par une pandémie ou connaître d'autres types de crises. La relocalisation de systèmes de production stratégiques pour la sécurité alimentaire du pays invite à revisiter les politiques d'innovation qui orientent les choix technologiques à long terme.

Le second niveau recommande d'intégrer l'approche «Une seule santé/One Health » dans la formulation des politiques agricoles et alimentaires. La crise actuelle rappelle l'incidence des zoonoses virales sur la sécurité sanitaire et les indicateurs de développement humain (espérance de vie...). Par ailleurs, si les forêts du bassin du Congo sont sources d'aliments comme la viande de brousse, elles sont aussi au centre de la préservation de la biodiversité mondiale (Görg et al., 2020). L'accélération de la déforestation dont elles font l'objet augmente les risques d'expression et de dissémination de maladies émergentes et zoonotiques.

L'agriculture vivrière et horticole (Kimbatsa et al., 2018) ainsi que l'élevage basés sur la production familiale, la transformation agro-artisanale et la distribution des produits sur les marchés physiques, devraient être plus soutenus par les 
politiques agricoles et alimentaires, et d'autant plus que ces activités génératrices de revenus concernent d'abord des populations vulnérables. Ces activités économiques structurent un système alimentaire socialement inclusif qui contribue à la lutte contre la pauvreté et à une alimentation saine et suffisante pour tous (Moustier, 2017). Mais pour construire ces nouvelles politiques, les décideurs doivent améliorer leur connaissance des besoins de développement des services d'appui, de recherche agronomique, de technologies et d'investissements en infrastructures, adaptés aux besoins locaux.

\section{Conclusion}

Au début de la crise sanitaire, les mesures de gouvernance publique pour maintenir fonctionnel le système alimentaire congolais au-delà des mesures de confinement général ont principalement veillé à: (i) assurer l'approvisionnement régulier des marchés; (ii) endiguer la flambée des prix des denrées de première nécessité; et (iii) prévoir la distribution des denrées de base aux populations les plus vulnérables et ayant perdu leur source de revenu. La gouvernance de cette crise, à court terme, par une flexibilité des mesures au regard de leurs incidences sur les flux de personnes et de biens assurant l'approvisionnement alimentaire, a favorisé l'adaptation rapide des acteurs. La pandémie a engendré une courte spéculation sur les denrées alimentaires, liée aux achats de panique, sans générer de pénurie ni de crise alimentaire majeure. Les systèmes de production et de distribution locaux ont réagi positivement. Nos analyses montrent que les variables qui expliquent la capacité de résistance du système alimentaire à la crise sanitaire reposent sur la flexibilité des mesures du gouvernement, l'agilité des acteurs alimentaires du secteur informel, l'usage des outils numériques et l'expérience liée aux crises antérieures qui avaient affecté les circuits alimentaires.

Les principales difficultés ont été enregistrées dans les filières (aviculture, aliment de bétail...) et dans les maillons (distribution, conservation, transformation et emballage) fortement liés aux intrants importés. Les villes ont été plus impactées que les milieux ruraux en raison de la concentration de la population et de l'activité économique, et du fait de leur double dépendance aux denrées alimentaires importées (viande, riz, farine de blé...) et à celles venant des zones rurales (manioc, fruit, viande de brousse...); seuls font exception les produits maraîchers produits en zone urbaine et péri-urbaine. Le besoin d'une plus grande autonomie du secteur agricole congolais par rapport aux marchés internationaux (en particulier pour la filière avicole et le maillon de la transformation) a été mis en exergue durant cette crise sanitaire. L'adaptation des investissements aux besoins du secteur agro-artisanal et domestique (à dominante informelle) est à rechercher dans le cadre d'une inflexion stratégique des politiques publiques qui devrait être alimentées en amont par des recherches spécifiques.

La gouvernance des mesures de gestion de la crise liée à la Covid-19 rencontre des difficultés dans la coordination des diagnostics, la définition des besoins prioritaires et des stratégies d'actions entre les différents ministères concernés par la santé et l'alimentation. Dans ce type de contexte, les décideurs publics doivent être en mesure de formuler des mesures d'urgences réalistes et de concevoir des politiques de long terme, les deux devant tenir compte des spécificités macro-économiques et écologiques du pays. La situation d'urgence révèle les composantes de résistance (agilité, expérience au vécu des crises, solidarité sociale, capacité endogène, usage du numérique, gouvernance réaliste), et donc des éléments de la résilience aux chocs, qui sont à soutenir dans le long terme. La crise de la Covid-19, en révélant la capacité d'adaptation des systèmes alimentaires locaux, crée de nouvelles conditions de connexions organisationnelles et technologiques entre la production agricole et l'approvisionnement des villes. Elle en révèle aussi les défaillances structurelles et le besoin d'accompagner les acteurs des filières par des politiques publiques adaptées et en mesure de réduire ces défaillances.

Cette crise interroge de manière concrète sur la pertinence de l'approche «One health» (Duru et al., 2017) dans la conception des politiques alimentaires et agricoles, notamment dans le contexte forestier du bassin du Congo, au regard du niveau de consommation de la viande de brousse et des causes possibles d'émergence de nouvelles pandémies. Cette conception impose d'élargir la connaissance des interactions entre les modèles de production agricoles et alimentaires d'une part, et la biodiversité des écosystèmes anthropisés ou peu connectés aux humains d'autre part. Le contexte de renouvellement du Plan national de développement (quinquennal) 2018-2022 et le processus des consultations infranationales en vue du Sommet des Nations unies sur les systèmes alimentaires de septembre 2021, offrent au Congo une opportunité d'ajustement de la réflexion collective, participative et inclusive de tous les acteurs du système alimentaire national, en vue de définir une politique agricole et alimentaire moins dépendante de l'extérieur et qui valorise mieux les ressources locales du pays.

\section{Références}

Bitsoumanou Nkounkou J, Martin G. 2020. L'impact de la crise du Covid-19 sur les systèmes alimentaires locaux du Congo et la réponse des institutions. Rome (Italy) : FAO. [2020/07/27] http:// www.fao.org/in-action/food-for-cities \%20 programme/news/ detail/en/c/1300543.

Bricas N, Tchamda C, Martin P. 2016. Les villes d'Afrique de l'Ouest et du Centre sont-elles si dépendantes des importations alimentaires? Cahiers Agricultures 25(5): 1-10. https://doi.org/10.1051/ cagri/2016036.

Courleux F. 2020. Ces lieux communs des débats agricoles à l'épreuve du Covid-19. Paysans \& société 3(381): 15-20. https:// doi.org/10.3917/pes.381.0015.

Duru M, Justes E, Falconnier G, Journet EP, Triboulet P, Magrini MB. 2017. Analyse du concept de santé globale pour accompagner les transitions agricoles et alimentaires : application au cas des légumineuses. Agronomie, Environnement \& Sociétés 7(1): 83-95.

Dury S, Alpha A, Zakhia-Rozis N, Giordano T. 2021. Les systèmes alimentaires aux défis de la crise de la Covid-19 en Afrique: enseignements et incertitudes. Cahiers Agricultures 30: 12. https:// doi.org/10.1051/cagri/2020052.

Elloumi M. 2020 L'agriculture tunisienne face à la Covid-19 : impacts de la crise sanitaire et perspectives pour une agriculture résiliente. Cahiers Agricultures 29: 35. https://doi.org/10.1051/cagri/ 2020032. 
FAO. 2020. Cities and local governments at the forefront in building inclusive and resilient food systems: Key results from the FAO Survey "Urban Food Systems and COVID-19". Revised version. Rome (Italy): FAO, 17 p. https://doi.org/10.4060/cb0407en.

Favrot M, Dorier E. 2018. Les politiques agricoles face au paradigme du sous-peuplement en République du Congo. Espace populations sociétés 3. https://doi.org/10.4000/eps.8135.

Feintrenie L. 2014. Agro-industrial plantations in Central Africa: risks and opportunities. Biodiversity and conservation 23(6): 1577-1589. https://doi.org/10.1007/s10531-014-0687-5.

Görg C, Plank C, Wiedenhofer D, Mayer A, Pichler M, Schaffartzik A, et al. 2020. Scrutinizing the Great Acceleration: the anthropocene and its analytic challenges for social-ecological transformations. The Anthropocene Review 1(7): 42-61. https:// doi.org/10.1177/2053019619895034.

INS. 2015. Projections et perspectives démographiques du Congo de 2007 à 2025. Brazzaville (République du Congo): INS, 44 p.

INS. 2021. Bulletin des statistiques du commerce extérieur du quatrième trimestre 2020. $\mathrm{N}^{\circ} 013$ /février. Brazzaville (République du Congo): INS, 18 p.

Kimbatsa FG, Mahoungou E, Ofouémé-Berton Y. 2018. L'importance de l'horticulture dans la lutte contre l'insécurité alimentaire, la pauvreté et la protection de l'environnement à Brazzaville (République du Congo). Études caribéennes 39-40. https://doi. org/10.4000/etudescaribeennes.12382.

Lepira FB, Mayamba Y, Nlandu JRRM, Kevaani R, Lepira DKT, Mangalaboyi JL. 2020. Lenteur de propagation de la Covid-19 en Afrique subsaharienne : réalité ou sommet de l'iceberg ? Cas de la République Démocratique du Congo. Annales Africaines de Médecine 13(3): 3759-3764.

Mbété RA, Banga-Mboko H, Ngokaka C, Bouckacka III QF, Nganga I, Hornick JL. 2011. Profil des vendeurs de viande de chasse et évaluation de la biomasse commercialisée dans les marchés municipaux de Brazzaville, Congo. Tropical Conservation Science 4(2): 203-217. https://doi.org/10.1177/194008291100400208.

Mfoukou-Ntsakala A, Bitémo M, Speybroeck N, Van Huylenbroeck G, Thys E. 2006. Agriculture urbaine et subsistance des ménages dans une zone de post-conflit en Afrique centrale. Biotechnologie, Agronomie, Société et Environnement 10 (3): 237-249.
Mialoundama Bakouétila GF, Ntoumbou Maboundou PL, Mbeté Roger A, Matoumona Mabiala NS, Bitsindou Kokolo HB, Missengué SS, et al. 2020. Impact de la Covid-19 sur la commercialisation de la viande de brousse: perception des commerçants de Brazzaville (Congo). International Journal of Progressive Sciences and Technologies 23(1): 53-62.

Moustier P. 2017. Short urban food chains in developing countries: signs of the past of the future? Natures Sciences Sociétés 25(1): 7-20. https://doi.org/10.1051/nss/2017018.

Murhula Balasha B, Kitsali Katungo JH, Mushagalusa Balasha A. 2021. Pauvreté et secteur informel à Bukavu: profil des détaillants des produits agricoles à l'ère de la pandémie Covid-19. Journal of Economics, Finance and Accounting Studies 3(1): 1-10. https:// doi.org/10.32996/jefas.2021.3.1.1.

Nations unies. 2020. Évaluation des impacts économiques et sociaux de la pandémie de Covid-19. Brazzaville (Congo) : Nations unies, 88 p.

Ofouémé-Berton Y. 1993. Identification des comportements alimentaires des ménages congolais de Brazzaville: Stratégie autour des plats. In : Muchnick J, ed. Alimentation, techniques et innovations dans les régions chaudes. Paris: 1'Harmattan, pp. 167-174.

Plan National de Développement. 2018-2022. Cadre stratégique de développement. République du Congo, 1, 194 p.

Sene I. 2020. Impact des mesures de prévention de la pandémie de Covid-19 sur les travailleurs du secteur informel au Sénégal. Revue Internationale du chercheur 1(4): 507-520.

Temple L, Rippke U, Pena J, Dury S. 2015. Identificación de los problemas alimentarios de la población vulnerable de Cali: análisis de las cadenas de abastecimiento y de producción. Cali Come Mejor. Reporte $n^{\circ} 4$. CIRAD Montpellier (France); CIAT Cali (Colombie), $48 \mathrm{p}$.

Vonthron S, Dury S, Fallot A, Alpha A, Bousquet F. 2016. L'intégration des concepts de résilience dans le domaine de la sécurité alimentaire : regards croisés. Cahiers Agricultures 25(6): 1-8. https://doi.org/10.1051/cagri/2016039.

Workie E, Mackolil J, Nyika J, Ramadas S. 2020. Deciphering the impact of Covid-19 pandemic on food security, agriculture, and livelihoods: A review of the evidence from developing countries. Current Research in Environmental Sustainability 2: 1-6. https:// doi.org/10.1016/j.crsust.2020.100014.

Citation de l'article : Bitsoumanou Nkounkou J, Temple L. 2021. Résilience du secteur alimentaire face à la crise Covid-19 et perspectives pour les politiques agricoles en République du Congo. Cah. Agric. 30: 39. 